\title{
Functional genomic analyses of Enterobacter, Anopheles and Plasmodium reciprocal interactions that impact vector competence
}

\author{
Nathan J. Dennison ${ }^{1 \dagger}$, Raúl G. Saraiva ${ }^{1 \dagger}$, Chris M. Cirimotich¹, Godfree Mlambo ${ }^{1}$, Emmanuel F. Mongodin² \\ and George Dimopoulos ${ }^{1 *}$ (i)
}

\begin{abstract}
Background: Malaria exerts a tremendous socioeconomic impact worldwide despite current control efforts, and novel disease transmission-blocking strategies are urgently needed. The Enterobacter bacterium Esp_Z, which is naturally harboured in the mosquito midgut, can inhibit the development of Plasmodium parasites prior to their invasion of the midgut epithelium through a mechanism that involves oxidative stress. Here, a multifaceted approach is used to study the tripartite interactions between the mosquito, Esp_Z and Plasmodium, towards addressing the feasibility of using sugar-baited exposure of mosquitoes to the Esp_Z bacterium for interruption of malaria transmission.
\end{abstract}

Methods: The ability of Esp_Z to colonize Anopheles gambiae midguts harbouring microbiota derived from wild mosquitoes was determined by qPCR. Upon introduction of Esp_Z via nectar feeding, the permissiveness of colonized mosquitoes to Plasmodium falciparum infection was determined, as well as the impact of Esp_Z on mosquito fitness parameters, such as longevity, number of eggs laid and number of larvae hatched. The genome of Esp_Z was sequenced, and transcriptome analyses were performed to identify bacterial genes that are important for colonization of the mosquito midgut, as well as for ROS-production. A gene expression analysis of members of the oxidative defence pathway of Plasmodium berghei was also conducted to assess the parasite's oxidative defence response to Esp_Z exposure.

Results: Esp_Z persisted for up to 4 days in the An. gambiae midgut after introduction via nectar feeding, and was able to significantly inhibit Plasmodium sporogonic development. Introduction of this bacterium did not adversely affect mosquito fitness. Candidate genes involved in the selection of a better fit Esp_Z to the mosquito midgut environment and in its ability to condition oxidative status of its surroundings were identified, and parasite expression data indicated that Esp_Z is able to induce a partial and temporary shutdown of the ookinetes antioxidant response.

Conclusions: Esp_Z is capable of inhibiting sporogonic development of Plasmodium in the presence of the mosquito's native microbiota without affecting mosquito fitness. Several candidate bacterial genes are likely mediating midgut colonization and ROS production, and inhibition of Plasmodium development appears to involve a shutdown of the parasite's oxidative defence system. A better understanding of the complex reciprocal tripartite interactions can facilitate the development and optimization of an Esp_Z-based malaria control strategy.

\footnotetext{
*Correspondence: gdimopo1@jhu.edu

${ }^{\dagger}$ Nathan J. Dennison and Raúl G. Saraiva contributed equally to this work

${ }^{1}$ W. Harry Feinstone Department of Molecular Microbiology

and Immunology, Bloomberg School of Public Health, Johns Hopkins

University, Baltimore, MD, USA

Full list of author information is available at the end of the article
} 
Keywords: Mosquito, Malaria, Microbiota, Transmission-blocking, Oxidative stress, Sugar-bait

\section{Background}

The impact of malaria transmission upon human health cannot be overstated, with up to two billion people worldwide at risk and an estimated 438,000 deaths yearly [1]. Plasmodium parasites, the causative agents of malaria, are transmitted through blood-feeding female anopheline mosquitoes. Plasmodium's complex life cycle provides multiple opportunities for intervention approaches, by targeting either the parasite or the mosquito vector. Strategies that target the parasite, including anti-malarial drugs, and those that target the vector, such as insecticides, are encountering resistance of the parasite and vector, respectively $[2,3]$. When mosquitoes bite a Plasmodium-infected person, the ingested Plasmodium gametocytes encounter a severe bottleneck in development at the ookinete stage within the midgut, the site where most parasites are killed $[4,5]$. During this infection stage, parasites also encounter the mosquito midgut microbiota. The negative impact of the mosquito microbiota on Plasmodium parasites has been well documented (reviewed in [6]) and offers an attractive opportunity to further narrow the bottleneck around Plasmodium development and its transmission between humans. The potential impact of the microbiota upon Plasmodium development suggests that studying these microorganisms could improve efforts at curtailing malaria transmission.

Interest in the tripartite interaction between vector, parasite, and microbiota has prompted the surveying of natural mosquito populations, revealing a microflora predominantly composed of Gram-negative species, with members of the Proteobacteria class often in high abundance [7, 8]. A number of naturally occurring bacteria have been shown to inhibit Plasmodium development in the mosquito, either through direct inhibition or indirectly through priming of basal immunity [9-11]. A correlation between $P$. falciparum infection and the prevalence of Enterobacteriacae has been documented [7], and several Gram-negative bacteria species have been shown to reduce the mosquitoes' vectorial capacity [1214]; also, a Chromobacterium species has been shown to possess anti-Plasmodium effects both in vivo and in vitro [15].

Screening isolates of the midgut microbiota of a Zambian mosquito population for Plasmodium-blocking activity has allowed the identification of an Enterobacter bacterium, Esp_Z, which is naturally harboured in the mosquito midgut tissue and can inhibit the development of Plasmodium parasites prior to their invasion of the mosquito midgut epithelium, independent of the mosquito's immune system [10]. Esp_Z nearly eliminates the development of malaria parasites to the human-infective sporozoite stage, thereby showing potential as a transmission-blocking agent.

$E s p_{-} Z$, even at low concentrations, is effective at inhibiting parasites in the mosquito intestine through a mechanism involving reactive oxygen species (ROS) [10]. Plasmodium parasites are susceptible to changes in the redox balance of their environment, and they defend themselves against ROS via an upregulation of antioxidant genes [16]. Two antioxidant systems with partially overlapping functions have been described in Plasmodium parasites, the thioredoxin and glutathione systems (reviewed in [17]). These systems are required to neutralize the highly oxidative environments encountered in both human erythrocytes [17] and the mosquito midgut [18]. The thioredoxin system is believed to be the main antioxidant system, since $P$. falciparum parasites lack a glutathione-dependent peroxidase $[19,20]$, suggesting that thioredoxin reduction of peroxiredoxin antioxidant enzymes plays a prominent role in protecting Plasmodium from toxic ROS [16]. Peroxiredoxin antioxidant enzymes such as peroxiredoxin-1 (Tpx-1) and 1-cysperoxiredoxin (1-Cys-prx) act as electron acceptors from thioredoxin-1 (Trx-1), maintaining them in a reduced state [21]. Plasmodium falciparum Tpx-1 knockout lines show an increased sensitivity to both ROS and reactive nitrogen species, but the deletion mutation is not lethal, suggesting redundancy in the parasite's antioxidant system [22]. A glutathione peroxidase-like thioredoxin peroxidase $\left(\mathrm{TPx}_{\mathrm{GI}}\right)$ has also been shown to be part of the thioredoxin system in Plasmodium and is reduced by Trx-1 [23]. Inhibitors of the thioredoxin pathway have been investigated as potential anti-malarial compounds, rendering the parasite more susceptible to oxidative stress [24].

Recently, reintroduction of several bacteria through the nectar meal, a natural route and vital mosquito energy source, has been demonstrated to inhibit Plasmodium sporogonic development, and methods for exposing mosquitoes to various agents through artificial nectars have been developed $[9,15,25]$. Manipulation of the composition of the mosquito microbiota may offer a strategy to reduce the prevalence of Plasmodium-infected mosquitoes and, therefore, malaria transmission. A multifaceted approach was used to test the feasibility of using sugarbaited exposure of mosquitoes to the $E s p_{-} Z$ bacterium for interruption of malaria transmission, by studying 
the reciprocal interactions between the bacterium and the mosquito and its microbiota, together with $E s p_{-} Z$ 's impact on the parasite under laboratory conditions. The $E s p \_Z$ genes that are likely to be conducive to effective colonization of the mosquito midgut and ROS-mediated parasite inhibition were specifically assessed, along with the effect of $E s p_{-} Z$ exposure on the parasite's oxidative defence system, mosquito fitness, and the ability of $E s p_{-} Z$ to compete with the endogenous bacteria of the mosquito midgut microbiota.

\section{Methods}

\section{Mosquito rearing and antibiotic treatment}

Anopheles gambiae Keele strain mosquitoes were maintained under laboratory conditions at $27{ }^{\circ} \mathrm{C}$ and $80 \%$ humidity with a $14 \mathrm{~h}$ day $/ 10 \mathrm{~h}$ night cycle. Larvae were reared on cat food pellets and ground fish food supplement. Adult mosquitoes were maintained on $10 \%$ sucrose and fed on mouse blood (mice were anesthetized with ketamine) for egg production. For clearance of the endogenous bacteria of laboratory mosquitoes, adult female mosquitoes were maintained on antibiotics immediately after eclosion $(75 \mu \mathrm{g} / \mathrm{mL}$ gentamicin sulfate [Quality Biological, Gaithersburg, MD, USA] and $100 \mu \mathrm{g} /$ $\mathrm{mL}$ penicillin-streptomycin [Invitrogen, Carlsbad, CA, USA] in a $10 \%$ sucrose solution ad libitum for three to 4 days). The sucrose-antibiotic solution was changed every $48 \mathrm{~h}$. To minimize the impact of any residual antibiotics, the mosquitoes were allowed to feed ad libitum for $24 \mathrm{~h}$ on a sterile $10 \%$ sucrose solution following antibiotic treatment.

\section{Bacteria cocktail preparation}

The bacterial cocktail was prepared from bacterial isolates previously identified from wild $A n$. arabiensis mosquitoes obtained through landing catches in Zambia [10]. Twelve species representing both Gram-negative and Gram-positive bacteria were selected. Single bacterial colonies from LB plates were selected and used to inoculate overnight cultures, then used to seed fresh cultures and grown to an $\mathrm{OD}_{600}$ of 1.0. Species names and GenBank sequence accession numbers were as follows: Knoellia sp. JF690939.1; Acinetobacter sp. JF690925.1; Bacillus sp. JF690926.1; Pseudomonas sp. JF690929.1; Exiguobacterium sp. JF690932.1; Kocuria sp. JF690933.1; Pantoea sp. JF690934.1; Pseudomonas sp. JF690935.1; Staphylococcus sp. JF690936.1; Arthrobacter sp. JF690937.1; Comamonas sp. JF690938.1; Bacillus sp. JF690930.1.

\section{Introduction of bacteria through sugar or blood meals}

Bacteria were grown in either LB or ookinete medium overnight at $30{ }^{\circ} \mathrm{C}$, used to seed fresh cultures, and then diluted to an $\mathrm{OD}_{600}$ of 1.0 , pelleted by centrifugation
(10 min, $3000 \mathrm{rpm}$ in a tabletop centrifuge), washed in $1 \times$ PBS, and finally resuspended in PBS. For sugarmeal introduction, bacteria were diluted in $3 \%$ sucrose to the concentration indicated in the text and provided to mosquitoes on moistened cotton strips. For bloodmeal introduction, mosquitoes were allowed to membrane-feed on blood containing bacteria (10\% bacterial solution, $40 \%$ blood, $50 \%$ human serum) at the concentration indicated in the text. Non-fed mosquitoes were removed within $24 \mathrm{~h}$ after blood-feeding.

\section{Anopheles gambiae midgut selection of Esp_Z}

The parental Esp_Z strain was provided to An. gambiae mosquitoes via blood meal at $10^{9} \mathrm{CFU} / \mathrm{ml}$ (P1). Engorged mosquitoes were maintained under standard insectary conditions, and 10 mosquitoes from this cohort were sampled daily for the presence of $E s p \_Z$ in the midgut. The $E s p \_Z$ bacteria that were found in the midgut for the longest time were then used to challenge a second mosquito population, and the process was repeated to isolate the "most fit" Esp_Z bacteria for midgut colonization (P2). This process of serial passage was then repeated a third time to isolate the midgut-selected $E s p_{-} Z$ bacteria (P3).

\section{Colonization experiments and DNA extraction}

To establish the ability of $E s p \_Z$ to colonize midguts harbouring a resident microbiota, $70-80$ female mosquitoes were treated with antibiotics as described above. Cocktail bacteria $\left(10^{6} \mathrm{CFU} / \mathrm{ml}\right)$ were introduced ad libitum for $48 \mathrm{~h}$ via sugar meal; the mosquitoes were then starved for 6-8 h, and Esp_Z $\left(10^{6} \mathrm{CFU} / \mathrm{ml}\right)$ was introduced by either blood-feeding or sugar meal. Every $24 \mathrm{~h}$, midguts were dissected from ten individual mosquitoes and transferred to a centrifuge tube containing $100 \mu \mathrm{lBS}$, then stored at $-80{ }^{\circ} \mathrm{C}$ for DNA extraction; three independent replicates were performed. Mosquitoes were surface-sterilized by washing them in $100 \%$ ethanol for 2 min and then rinsing them for $1 \mathrm{~min}$ in sterile $1 \times$ PBS. DNA extractions were carried out as previously described [26] with minor modifications: In brief, dissected midguts were homogenized in $90 \mu \mathrm{l}$ PBS, followed by the addition of $90 \mu \mathrm{l}$ lysozyme $(40 \mathrm{mg} / \mathrm{ml})$ and incubation at $37{ }^{\circ} \mathrm{C}$ for $1 \mathrm{~h}$; $300 \mu \mathrm{l}$ of extraction buffer ( $1 \%$ SDS; $50 \mathrm{mM}$ Tris- $\mathrm{HCl}$, pH 8.0; $25 \mathrm{mM} \mathrm{NaCl} ; 25 \mathrm{mM}$ EDTA, pH 8.0) was added to samples and incubated at $65{ }^{\circ} \mathrm{C}$ for $10 \mathrm{~min}$. Following the addition of $200 \mu \mathrm{l}$ of $3 \mathrm{M}$ potassium acetate ( $\mathrm{pH}$ 7.2), samples were incubated on ice for $1 \mathrm{~h}$ and then centrifuged at $14,000 \mathrm{rpm}$ in a tabletop centrifuge for $10 \mathrm{~min}$ and the supernatants removed. The pellets were then treated with RNase $(40 \mathrm{mg} / \mathrm{ml})$ at room temperature for $30 \mathrm{~min}$. The supernatants were extracted twice with phenol/chloroform/isoamyl alcohol and precipitated with 2.5 volumes 
of $100 \%$ ethanol and 1:10 $3 \mathrm{M}$ sodium acetate at $-20{ }^{\circ} \mathrm{C}$ overnight; the pellets were resuspended in $10 \mu \mathrm{l}$ of water.

\section{Absolute qPCR quantification}

All mosquito tissues were stored at $-80{ }^{\circ} \mathrm{C}$ following dissection, and DNA was extracted as described. Absolute qRT-PCR determination was used to determine total bacteria and Esp_Z gene copy numbers. Degenerate primers targeting the $16 \mathrm{~S}$ rRNA sequence of each bacterium were designed from previously sequenced 16S rRNA gene fragments, following Clustal W nucleotide alignment of the sequences [10]. Primers targeting Esp_Z gene sequences were designed from $E s p_{-} Z$ genomic scaffolds. Primers were checked for potential cross-hybridization through BLASTn searches against the nr gene database, retrieving no significant hits $(E$ value $=0.1) . E s p \_Z$ primers were tested for specificity for the bacterium through PCR amplification from DNA of An. gambiae midguts fed the bacterial cocktail species. PCR fragments used for standard curves were cloned using the pGEM-T Easy vector (Promega). Standard curves for $16 \mathrm{~S}$ rRNA and $E s{ }_{-} Z$ had efficiencies between 80 and $100 \%$ and $\mathrm{R}^{2}>$ 0.99 . The relative proportion of $E s p_{-} Z$ was calculated by dividing the total number of $E s p_{-} Z$-specific copies by the number of amplified 16S rRNA copies across three biological replicates. Amplification and detection of bacterial DNA was performed using the QuantiTect SYBR Green PCR Kit (Qiagen, Valencia, CA, USA) and ABI Detection System ABI Prism 7000, using two technical replicates. The PCR reaction was performed in a total volume of $20 \mu \mathrm{l}$, which consisted of $2 \times$ Sybr green master mix (Applied Biosystems, Foster City, California, USA), $75 \mathrm{nM}$ of each primer, and $50 \mathrm{ng}$ of template DNA. The cycling parameters were as follows: initial denaturation at $95{ }^{\circ} \mathrm{C}$ for $2 \mathrm{~min}$, followed by 40 cycles of $95^{\circ} \mathrm{C}$ for $15 \mathrm{~s}$, $56^{\circ} \mathrm{C}$ for $30 \mathrm{~s}$, and $60^{\circ} \mathrm{C}$ for $30 \mathrm{~s}$. Following amplification, a melting curve analysis was performed from 60 to $95^{\circ} \mathrm{C}$, collecting fluorescence data every $0.5^{\circ} \mathrm{C}$.

\section{Relative $\mathrm{qPCR}$ quantification}

To conduct relative real-time qPCR assays, RNA was extracted using TRIzol $^{\circledR}$ (Life Technologies, Carlsbad, CA) according to the manufacturer's guidelines and treated with Turbo DNase; first-strand cDNA was produced using Superscript III reverse transcriptase (Invitrogen). cDNA templates were normalized to the Plasmodium berghei $18 \mathrm{~S}$ rRNA gene as previously described [16], and fold changes in gene expression levels were determined using the standard [27]. Assays were performed using Sybr green master mix with the following cycling parameters: initial denaturation at $95^{\circ} \mathrm{C}$ for $2 \mathrm{~min}$, followed by 40 cycles of $95{ }^{\circ} \mathrm{C}$ for $15 \mathrm{~s}, 56{ }^{\circ} \mathrm{C}$ for $30 \mathrm{~s}$, and $72{ }^{\circ} \mathrm{C}$ for $30 \mathrm{~s}$.

\section{Longevity, fecundity, and fertility assays}

To assess the impact of bacterial introduction on mosquito longevity, bacteria were introduced into aseptic female mosquitoes, and the impact of $E s p \_Z$ was compared to that of the introduction of the bacterial cocktail or a PBS-only control. For each treatment, approximately 60 female mosquitoes were rendered free of their microbiota through antibiotic treatment, and then either Esp_Z, the bacterial cocktail, or PBS was introduced by either a blood or sugar meal. Each cohort was then provided with a naïve blood meal at 4 days post-introduction. Non-fed mosquitoes were removed and excluded from the analysis. Mosquito mortality was monitored daily; monitoring continued until all the mosquitoes had perished, and survival percentages were calculated across three biological replicates. Kaplan-Meier survival analysis was carried out using GraphPad Prism 5 software, and $p$ values were determined by log-rank test (Mantel-Cox) and corrected for multiple comparisons by the Bonferroni method.

For the fecundity and fertility experiments, 30-60 female mosquitoes were treated with antibiotics as described, and three treatment groups were established with $E s p \_Z$, the bacterial cocktail, and PBS being introduced by both sugar and blood meals. Following bloodmeal introduction, blood-fed mosquitoes were removed to individual vials $(12 \mathrm{ml})$ lined with moistened filter paper and then incubated under normal rearing conditions. Following sugar-meal introduction, mosquitoes were provided a naïve blood meal $48 \mathrm{~h}$ later, and the fed mosquitoes were removed to individual vials. Eggs oviposited on the filter paper were counted daily using a light microscope for 2 days. Females that did not produce eggs by day 2 were re-examined on day 3 . After counting, eggs were submerged in water under standard larval rearing conditions, and the hatch rate (fertility) was determined by counting first instar larvae, which were then removed daily. Three biological replicates were performed for the fecundity and fertility assays and significance was determined using the Kruskal-Wallis test.

\section{Plasmodium infection assays}

Mosquitoes were fed on NF54 P. falciparum gametocyte cultures $(0.01 \%$ gametocytaemia; provided by the Johns Hopkins Malaria Institute Parasitology Core Facility) through artificial membranes at $37^{\circ} \mathrm{C}$. The adult mosquitoes were starved for $8-12 \mathrm{~h}$ prior to feeding to ensure engorgement, and unfed mosquitoes were removed from the cohort within $24 \mathrm{~h}$. To determine oocyst numbers, the mosquitoes were incubated for a further 7 days at $27{ }^{\circ} \mathrm{C}$, and midguts were dissected out in PBS, stained with $0.2 \%$ mercurochrome, and examined using a lightcontrast microscope (Olympus). To establish whether 
Esp_Z is capable of inhibiting Plasmodium development when provided via sugar meal, 50-70 female septic mosquitoes were reared as described above and then provided a sugar meal containing Esp_Z $Z$ at varying concentrations for 3-4 days; they were then provided a P. falciparum-infected blood meal the following day. Oocyst numbers were determined as described above and compared to a cohort fed only PBS containing $3 \%$ sucrose.

\section{Esp_Z genome sequencing and transcriptome analysis}

Total bacterial DNA was extracted from an Esp_Z liquid culture. A $3 \mathrm{~kb}$ insert paired-end shotgun genomic library was prepared from the extracted DNA and sequenced using the 454 GS FLX Titanium sequencing platform at the Genome Resource Center at the Institute for Genome Sciences (IGS) of the University of Maryland according to the manufacturer's protocol. Sequencing reads were then assembled using Celera Assembler, and ORFs were predicted and annotated using the IGS Annotation Engine implemented within the CLoVR-Microbe pipeline [28]. Total bacterial RNA from bacterial liquid cultures grown in either LB or ookinete medium was extracted using TRIzol according to the manufacturer's protocol, treated with Turbo DNase (5U, Life Technologies), cleaned using an RNeasy Mini Kit (Qiagen, Valencia, CA, USA), and the quality determined by an Agilent Bioanalyzer 2100. A custom $8 \times 44 \mathrm{~K}$ Agilent microarray was designed based on the 454-generated Esp_Z genome sequence. Cy-3- or Cy-5-labeled cRNA probes was synthesized from $300 \mathrm{ng}$ of total RNA per replicate using the LabelIT ${ }^{\circledR}$ MicroArray Dual Labeling Kit (Mirus Bio, Madison, WI, USA). Labelled RNA was hybridized overnight at $65^{\circ} \mathrm{C}$, and arrays were scanned with an Agilent scanner. Transcript abundance data were processed and analysed as previously described [29, 30]. In brief, LOWESS normalized background-subtracted median fluorescent values were used to determine $\mathrm{Cy} 5 / \mathrm{Cy} 3$ ratios from replicate assays and were then subjected to $t$ tests at a significance level of $p<0.05$ using MIDAS, GEPAS, and TMEV software [31, 32]. The rate for self-self hybridizations was used to calculate a cut-off value for the significance of gene regulation on these microarrays of $\log 2 \mathrm{FC}=0.78$ or 1.72 -fold regulation [33].

\section{In vitro parasite culture and co-culture with bacteria}

For the rodent malaria parasite production, cultures were carried out as previously described [10] with certain modifications. In brief, $P$. berghei GFP parasites were injected into donor Swiss Webster mice and monitored daily for parasitaemia for 3 days postinfection. Once parasitaemia reached $>15 \%$, infected blood was collected by heart puncture and transferred to phenylhydrazine-treated mice. Mice were treated with pyrimethamine $72 \mathrm{~h}$ later and monitored daily for parasitemia and exflagellation. Parasitized mice with $\geq 20$ exflagellation events per $20 \times$ microscope field were used for in vitro experiments. Parasitized blood was collected by heart puncture and diluted 1:10 in ookinete medium ( $10 \%$ foetal bovine serum, $50 \mu \mathrm{g} / \mathrm{ml}$ hypoxanthine, $2 \mathrm{mg} / \mathrm{ml} \mathrm{NaHCO}, 1 \mu \mathrm{M}$ xanthurenic acid in RPMI 1640 medium, pH 8.3), 4 \% RBC lysate, and experiment-specific constituents in a $500 \mu \mathrm{l}$ total volume. To assess the induction of $P$. berghei antioxidant genes, bacteria (final concentration $10^{6} \mathrm{CFU} / \mathrm{ml}$ ) were added to the ookinete culture at $0 \mathrm{~h}$. Culture samples were directly transferred to Tri-Reagent (Ambion) at various times, from 1 to $10 \mathrm{~h}$ after setup, for total RNA extraction. First-strand cDNA was then prepared for qPCR analysis. Development of ookinetes was confirmed by Giemsa staining at $24 \mathrm{~h}$.

\section{Results and discussion}

Colonization of the Anopheles gambiae midgut by Esp_Z

$E s p_{-} Z$ has been detected within the midguts of fieldcaught anopheline malaria vectors [10], but its capacity to persist within the mosquito following artificial introduction is not known. The extent to which Esp_Z can colonize the mosquito midgut, the site of Plasmodium pre-oocyst development, is likely to contribute to the magnitude of the Plasmodium inhibition. The persistence of $E s p_{-} Z$ in the mosquito midgut when co-present with a simulated natural microbiota was determined. The midgut microflora of field-caught mosquitoes has been shown to differ markedly from its laboratory-reared counterparts [7, 34]; therefore, a cocktail of bacteria previously isolated from the same southern Zambian populations of $A n$. arabiensis from which $E s p \_Z$ was identified was utilized [10]. To determine the relative abundance of $E s p \_Z$ over time, the ratio of $E s p \_Z$ to total bacterial $16 \mathrm{~S}$ rRNA was calculated through quantitative real-time PCR. Two approaches were taken to introduce $E s p \_Z$ into mosquitoes already harbouring a resident microbiota: either through a blood meal as shown before for this bacterium [10] or through a sugar meal to inquire on the viability of introducing $E s p_{\_} Z$ to anophelines via nectar feeding. Esp_Z $Z$ bacteria were able to persist within the midguts of cocktail-colonized mosquitoes following blood-meal introduction for up to 72 h. Esp_Z constituted an average $32.8,48.5$, and $30.3 \%$ of the total bacterial population at 24,48 , and $72 \mathrm{~h}$ post-introduction, respectively. Esp_Z bacteria were detected up to $120 \mathrm{~h}$ post-introduction but reflected $<1 \%$ of the total microbiota (Fig. 1a, top). Following the introduction of Esp_Z through sugar feeding into mosquitoes already harbouring a cocktail microbiota, Esp_Z bacteria were detected for 4 days, peaking at an initial $11.1 \%$ of the total 
microbiota following introduction and still detectable (3.5\%) at day 4 (Fig. 1a, bottom).

Although the bacterial cocktail consisted of strains isolated from field mosquitoes, the microflora of individual mosquitoes is highly variable [7]. Therefore, the persistence of $E s p \_Z$ for 4 days in the presence of natural microflora is encouraging. It also indicates that, when possible, a bacterium destined for field-based release must be investigated in the context of a natural microbiota. In addition, acquisition of a sugar meal provides energy reserves for flight [35] and promotes mosquito longevity $[25,36]$, suggesting that multiple sugar meals may be taken during the mosquitoes' lifespan. Note that when introduced through the sugar meal, Esp_Z tended to constitute a lower proportion of the total microbiota when compared to ingestion by blood-feeding (Fig. 1a). In the field, it is estimated that Anopheles mosquitoes feed on blood every two or 3 days [37, 38]; knowing there is a rapid expansion of the gut microbiota upon blood intake $[39,40]$, it is reasonable to assume that Esp_Z, even when introduced by nectar feeding, could persist in the mosquito gut beyond what was observed in a laboratory setting. Therefore, when considering a field-based application of bacteria, sugar-meal introduction would be best evaluated in the context of the specificities of the anophelines and their feeding behaviour in any given ecological niche. In summary, it is shown that Esp_Z can be introduced through both bacteria-enriched blood and sugar sources and persist for multiple days in competition with a natural microbiota.

\section{Inhibition of Plasmodium sporogonic development following sugar-meal introduction of Esp_Z}

Having determined that $E s p_{-} Z$ can persist within the mosquito midgut for up to 4 days following sugar-meal introduction, the ability of $E s p_{-} Z$ to inhibit Plasmodium development when provided to mosquito cohorts through a sugar meal was investigated next. Previous experiments have shown $E s p_{-} Z$ is highly effective at inhibiting Plasmodium development at the ookinete stage when provided in a blood meal [10]. Use of attractive toxic sugar baits has proven successful in reducing mosquito populations [41], indicating that bacteria, in this context, could be disseminated through sugar feeding. To simulate an approximation of how $E s p \_Z$ bacteria would be disseminated through a natural population, mosquitoes, containing their endogenous microflora, were provided $E s p_{-} Z$ suspended in
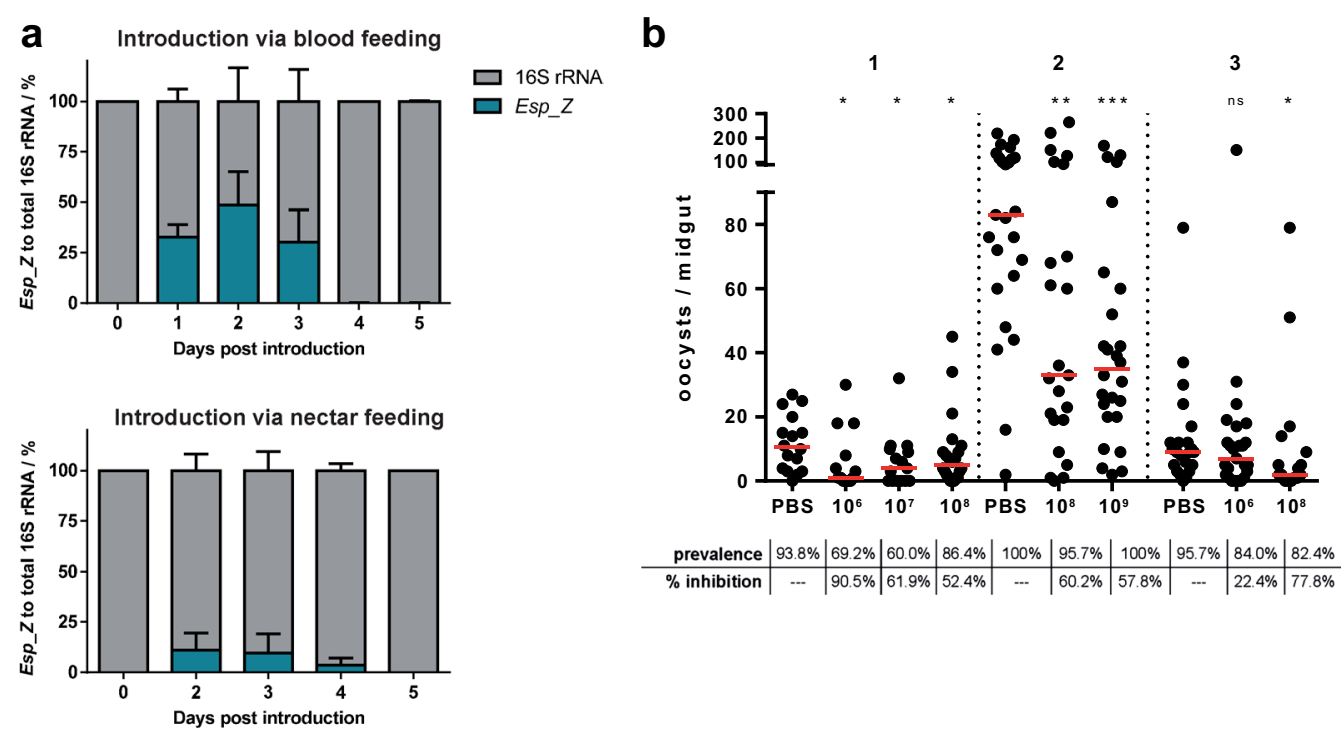

\begin{tabular}{|c|c|c|c|c|c|c|c|c|c|c|}
\hline inhibition & --- & $90.5 \%$ & $61.9 \%$ & $52.4 \%$ & -- & $60.2 \%$ & $57.8 \%$ & -- & $22.4 \%$ & $77.8 \%$ \\
\hline
\end{tabular}

Fig. 1 Colonization of the Anopheles gambiae midgut by Esp_Z and impact on Plasmodium falciparum sporogonic development. a Esp_Z was introduced into a mosquito cohort already containing a cocktail of naturally occurring bacteria through either a blood or sugar meal, and DNA extracted from ten midguts was sampled daily. The Esp_Z to $16 \mathrm{~S}$ rRNA ratio was determined using Esp_Z-specific and universal 16S rRNA standard curves. b Female An. gambiae containing their endogenous microflora were provided with PBS or with Esp_Z at the indicated concentration (10x CFU/mL) suspended within $3 \%$ sucrose. After 3 to 4 days of being allowed to feed on these suspensions, they were given a P. falciparum-infected blood meal, and oocyst numbers were determined 7 days later. Oocyst counts for three independent replicates are shown (Rep 1-3). Horizontal bars represent the median number of oocysts per treatment; inhibition (\%) was estimated based on the comparison of these values to that of the PBS control. Prevalence represents the proportion of infected mosquitoes per group. Significance was determined using the Mann-Whitney test by comparing treatment groups to their respective control cohorts $\left({ }^{*} p<0.05 ;{ }^{* *} p<0.01 ;{ }^{* * *} p<0.001\right)$ 
$3 \%$ sucrose and allowed to feed for three to 4 days. Plasmodium infection assays revealed that the impact of $E s p \_Z$ upon parasite development when provided through a sugar meal averaged a $45-65 \%$ inhibition across the different concentrations tested (Fig. 1b). Of note, however, is the inter-replicate variability that was observed, especially at the lowest tested concentration of $10^{6} \mathrm{CFU} / \mathrm{mL}$; inhibition varied from $22.2 \%$ in one replicate (from May 2014) to $90.5 \%$ in another (February 2014). Seasonal variations in the mosquitoes' resident microbiota may provide an explanation for these results. In particular, the midguts of mosquitoes fed in May 2014 were dominated by a different bacterium as observed by the colonies that resulted of plating ground midguts in LB agar. It is also likely that individual mosquitoes actually fed on the Esp_Z sucrose solution at different times prior to the infected blood meal, suggesting that they would harbour different $E s p \_Z$ concentrations and explaining how these results might differ from the ones obtained when $E s p \_Z$ was introduced directly with the blood meal [10]. Within $24 \mathrm{~h}$ after mosquito ingestion of Plasmodium gametocytes, the number of developing ookinetes is substantially lower than the initial gametocyte number [42], and this bottleneck occurs as the result of numerous factors [5]. Therefore, if detectable numbers of $E s p_{-} Z$ are present $24 \mathrm{~h}$ after an infectious blood meal, they are likely to further constrict this bottleneck and reduce Plasmodium transmission.

\section{Esp_Z influence on mosquito fitness parameters}

It has previously been shown that the reintroduction of naturally occurring bacteria can affect mosquito fitness [9]. Recently, a Chromobacterium species has been identified that displays both anti-parasitic and entomopathogenic properties and may therefore be used to reduce both disease transmission and vector populations [15]. To provide insight into the impact of Esp_Z bacteria on An. gambiae physiology, its effects on mosquito longevity, fecundity, and fertility were assayed following introduction via the two methods, a blood meal and continuous sugar feeding. To assay longevity, Esp_Z, the bacterial cocktail, or a bacteria-free PBS control were introduced into aseptic mosquitoes and monitored mortality daily. Following introduction via sugar-feeding, there was no significant difference between the mosquitoes harbouring no bacteria (aseptic) and those continuously fed $E s p \_Z$ ( $p$ value $\left.=0.3852\right)$, or those harbouring the bacterial cocktail and those fed to $E s p \_Z$ ( $\left.p>0.9999\right)$, all having a median survival of 16 days (Fig. 2a). Conversely, when introduced through the blood meal, Esp_Z had a significant impact on longevity when compared to the bacterial cocktail $(p=0.0004)$ and to the aseptic group $(p=0.0308)$ (Additional file 1: Figure S1A). The presence
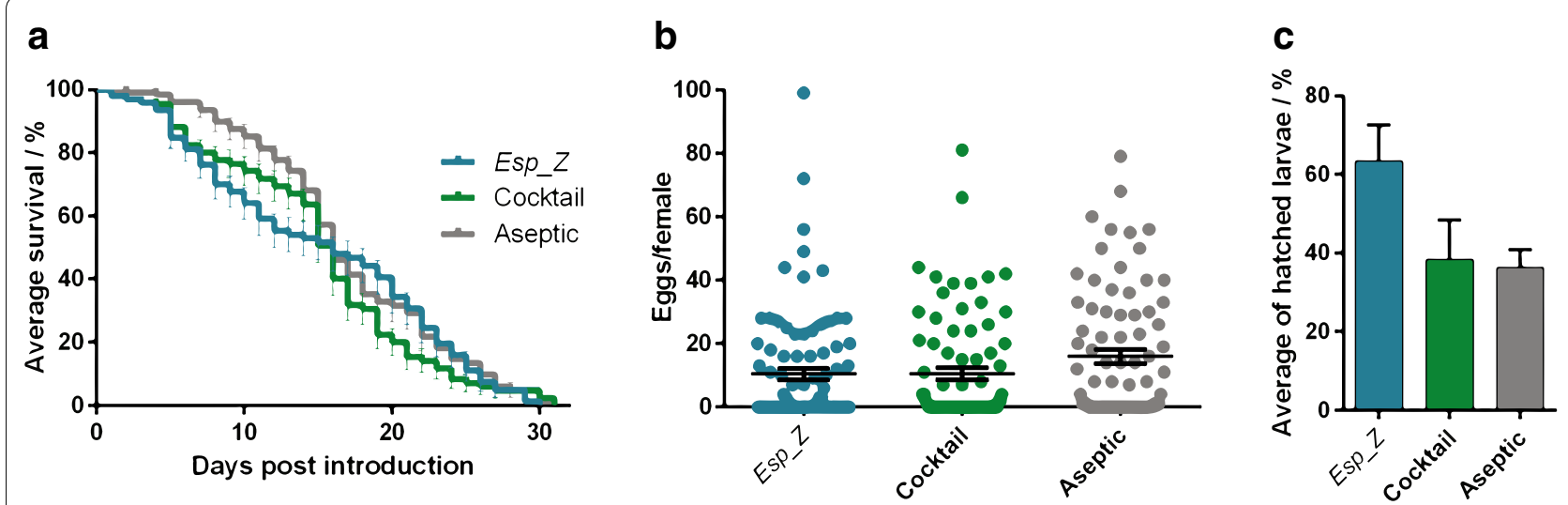

Fig. 2 Sugar-meal introduction of Esp_Z has no impact on Anopheles gambiae fitness. a Longevity studies were performed following continuous sugar-meal introduction of either Esp_Z, a bacterial cocktail, or PBS into aseptic mosquito cohorts. A sterile blood meal was provided on day 4 (black arrow), and unfed mosquitoes were censored from the analysis. Survival was monitored daily and continued until $100 \%$ mortality was reached. The curves represent the average percent mortality across three replicates; the error bars indicate standard error. Significance was determined using the log-rank test (Mantel-Cox) with Bonferroni correction using a Kaplan-Meier survival analysis. Esp_Z vs Cocktail, $p>0.9999$; Esp_Z vs Aseptic, $p=$ 0.3852. b Comparative fecundity analysis of the Esp_Z-, bacterial cocktail- and PBS-fed, sugar-fed mosquito cohorts. Separate cohorts were provided with a blood meal $72 \mathrm{~h}$ after introduction, and circles represent the number of eggs laid per female. Horizontal bars represent the median number of eggs, and error bars indicate the standard error; three pooled biological replicates are shown. $p=0.0596$ (Kruskal-Wallis test). c Fertility analysis in Esp_Z-, bacterial cocktail-, and PBS-fed mosquitoes. At $72 \mathrm{~h}$ after introduction, mosquitoes were offered a blood meal, and those not engorged were removed. Eggs were collected at $48 \mathrm{~h}$ post-blood meal and allowed to hatch in rearing trays. The hatch rate indicates the percentage of eggs giving rise to 1st instar larvae; the error bars indicate the standard error of the mean. $p=0.0741$ (Kruskal-Wallis test) 
of Esp_Z, introduced by either method, did not significantly affect egg production (via sugar meal, Fig. $2 \mathrm{~b}$, $p=0.0596$; via blood meal, Additional file 1: Figure S1B, $p=0.0507)$ nor mosquito fertility measured by hatched larvae (via sugar meal, Fig. 2c, $p=0.0741$; via blood meal, Additional file 1: Figure S1C, $p=0.1000$ ).

There was no impact of $E s p_{-} Z$ on mosquito survival when provided in a sugar meal (Fig. 2a), whereas blood-feeding significantly negatively affected mosquito longevity (Additional file 1: Figure S1). This result is perhaps expected, since acquisition of the native microflora likely occurs through sugar meals $[39,40]$, whereas blood-meal introduction is an artificial system and would result in a sudden expansion of the midgut microbiota. Taken together, these data suggest that in a field-based approach, Esp_Z introduced through sugarbaited traps would not adversely affect mosquito fitness and, therefore, the presence of $E s p \_Z$ would not be selected against when artificially reintroduced into field mosquitoes.

\section{Esp_Z genome analysis}

To gain insight into the genetic basis of the Esp_Zmediated inhibition of Plasmodium and develop tools for bacterial functional genomics, the $E s p \_Z$ genome was sequenced via a whole-genome shotgun sequencing approach, as described in "Methods" section. A total of 864,207 Titanium sequencing reads were obtained, corresponding to an approximately $38 \times$ genome coverage. After assembly and ORF prediction, a total of 39 scaffolds were obtained, two of which were identified as plasmidrelated through identity with known plasmid sequences in GenBank. PCR and Sanger-based sequencing analysis confirmed the presence of two closed plasmids. The estimated size of the chromosomal Esp_Z genome is then $5.2 \mathrm{Mbp}$, with a GC-content averaging $55.8 \%$ (Fig. 3a). A phylogenetic analysis based on $16 \mathrm{~S}$ rRNA genomic sequences allowed for the confirmation of $E s p \_Z$ as a novel Enterobacter species (Fig. 3b). The Esp_Z genome contains 4919 putative protein-coding genes that were subjected to SEED functional analysis (Fig. 3c) and used
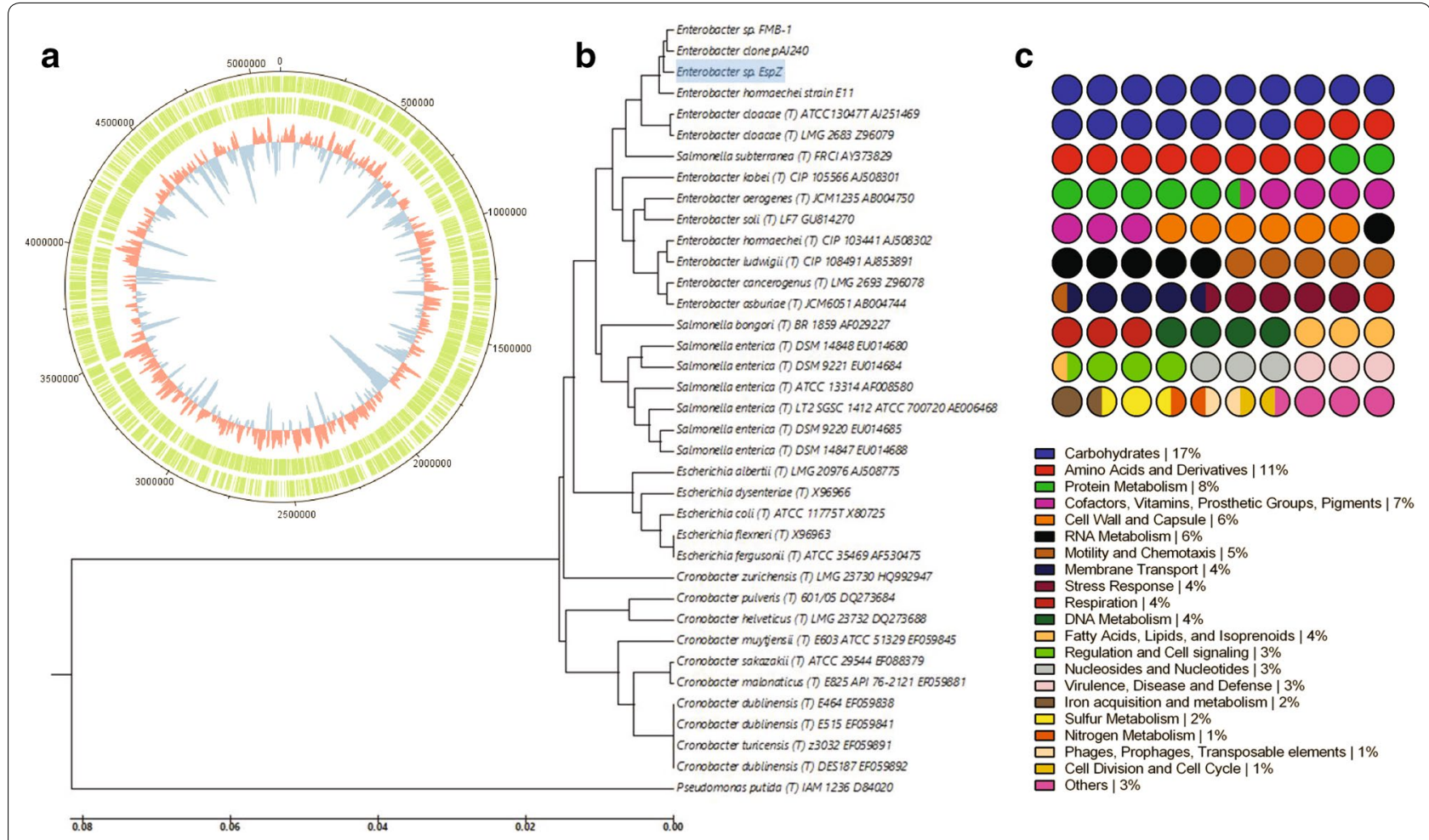

Fig. 3 Esp_Z genome. a Circular representation of the Esp_Z genome. The coding regions of both the forward and reverse strands are shown in green; blue represents a negative deviation and red a positive deviation of the average G-C content for this genome of $55.8 \%$. b Evolutionary relationships between Esp_Z and related type and non-type strains, based on 16S rRNA genomic sequences and inferred by the UPGMA method. The optimal tree with the sum of branch lengths equal to 0.33591652 is shown. The tree is drawn to scale, with branch lengths in the same units as those of the evolutionary distances used to infer the phylogenetic tree. The evolutionary distances were computed using the maximum composite likelihood method and are expressed as the number of base substitutions per site. The analysis was conducted in MEGA6 and involved $37 \mathrm{genomic}$ sequences. c Distribution of the functional role categories of the protein-encoding genes of Esp_Z using the SEED database; $47 \%$ of those genes were not assigned a functional role and are omitted from this representation 
to construct microarrays for whole-genome expression analysis.

\section{Transcriptomic survey of Esp_Z genes that mediate selection in the mosquito midgut environment}

To enhance the process of Anopheles midgut colonization, the $E s p \_Z$ bacterium was serially passaged and selected for prolonged persistence in the mosquito midgut, as described previously [43]. The persistence of the parental $E s p_{-} Z$ strain was able to increase from four to eight days by selecting for bacteria best able to survive in the mosquito gut (Fig. 4a). A total of 41 genes were differentially regulated between the parental (P1) and passaged (P3) Esp_Z strains, 20 genes with increased expression in the passaged strain and 21 in the parental (Fig. 4b; Additional file 2: Table S1).

Of particular interest within the 20 regulated genes of the passaged bacterium were a type III secretion apparatus protein, a sugar transport component, a glutathione S-transferase, and an oxidoreductase (Table 1). Type III secretion systems have been shown to modulate eukaryotic host immune cell signalling, allowing colonization of the host $[44,45] . E s p \_Z \_3940(\log 2 \mathrm{FC}=1.07)$ showed the highest similarity to Pantoea sp. Type III secretion system export protein SpaR/YscT/HrcT, and a Pantoea Type III secretion has been shown to be important for bacterial persistence in an insect vector, the flea beetle Chaetocnema pulicaria [46]. A sugar $\mathrm{ABC}$ transporter component was also found in increased abundance in the passaged strain $(\log 2 \mathrm{FC}=0.83)$. A component of the sugar transport system has previously been identified as a key virulence factor in bacterial colonization [47], and during Bacillus cereus colonization of the honeycomb moth, a sugar sensing and transport operon is upregulated [48]. Because sugars and glucosidases are present in the mosquito midgut, it is possible that $E s p_{-} Z$ adapts to its host environment through increased expression of sugar transport genes to allow the utilization of available nutrients. Following ingestion of a blood meal, the mosquito midgut environment is rich in haem, which can modulate the oxidative state of the midgut environment [49]. An increased abundance of GST $(\log 2 \mathrm{FC}=0.88)$ and oxidoreductase $(\log 2 \mathrm{FC}=0.84)$ transcripts in the selected bacterium was observed, suggesting that the
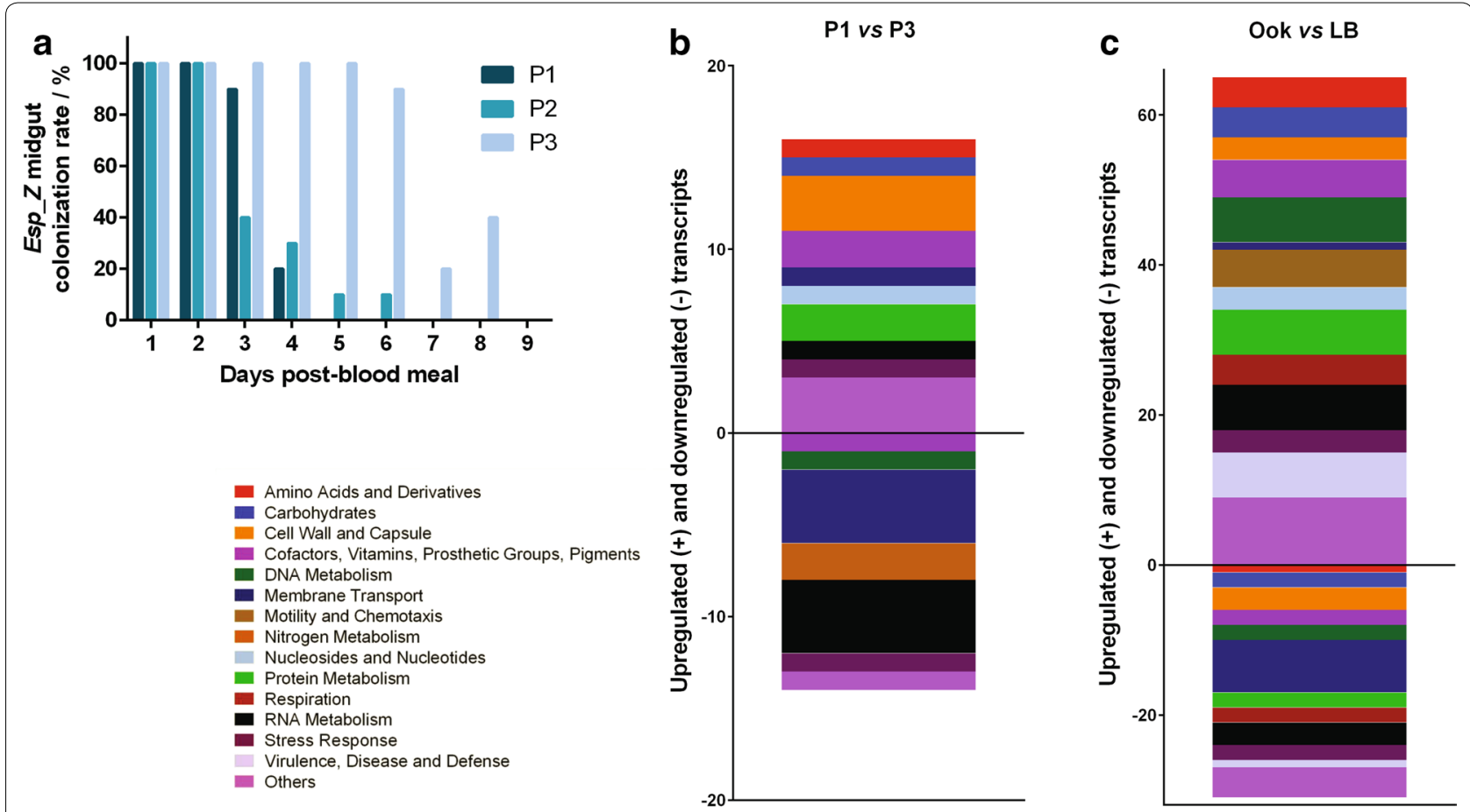

Fig. 4 Differential gene expression in Esp_Z on different culture media and upon serial passage in the Anopheles gambiae midgut. a A parental strain of Esp_Z (P1) was provided to An. gambiae mosquitoes through a blood meal and sampled daily. Colonies persisting for the longest time were reintroduced into a second cohort (P2) and the process repeated a third time to isolate the passaged (P3) strain. The ability of a selected strain of Esp_Z to persist within the An. gambiae midgut was determined through qRT-PCR copy number analysis. $\mathbf{b}$ Distribution of the functional role category of upregulated $(+)$ and downregulated $(-)$ transcripts of parental P1 when compared to passaged P3. Functional role categories were assigned to 59/98 upregulated and 29/61 downregulated transcripts using the SEED database. c Distribution of the functional role category of upregulated (+) and downregulated ( - ) transcripts of Esp_Z grown in ookinete medium when compared to LB medium. Functional role categories were assigned to 15/21 upregulated and 14/20 downregulated transcripts using the SEED database 
Table 1 Colonization adaptation-related candidate genes that are differentially regulated between the parental (P1) and adapted (P3) Esp_Z strains

\begin{tabular}{llll}
\hline Gene ID & Description & Process GO category & Fold change $\left(\mathbf{l o g}_{\mathbf{2}}\right.$ ) \\
\hline \multicolumn{2}{l}{ Parental upregulated } & & 0.999 \\
ESP_Z_1310 & yadA-like C-terminal region family protein & N/A & 0.897 \\
ESP_Z_1067 & SOS cell division inhibitor & SOS response & 0.834 \\
ESP_Z_3679 & uxuR transcriptional repressor & Regulation of transcription, DNA-dependent \\
Adapted upregulated & & 1.065 \\
ESP_Z_2042 & Type III secretion apparatus protein & Transport & 0.878 \\
ESP_Z_4047 & Glutathione S-transferase, C-terminal domain protein & Metabolic process & 0.836 \\
ESP_Z_0990 & Oxidoreductase FAD-binding domain protein & Oxidation-reduction process & 0.827 \\
ESP_Z_4206 & Sugar abc transporter permease & Transport & \\
\hline
\end{tabular}

passaged strain is able to elevate its response to the harsh ROS rich environment in order to maintain redox homeostasis and persist within the mosquito midgut. Sugarfed Aedes aegypti mosquitoes constitutively express ROS in the midgut, so it is possible that the selected bacterium elevates antioxidant genes in both the sugar- and bloodfed environments [50].

Amongst the genes with increased expression in the parental Esp_Z strain (Table 1), YadA transcripts ( $\log 2 \mathrm{FC}=1.0)$ are of particular note. YadA is a bacterial adhesin able to mediate binding to epithelial cells $[51,52]$ and may putatively align Esp_Z in close proximity to the mosquito midgut epithelium and result in elicitation of immune response [53]. Decreased YadA transcription in the passaged strain may therefore have the potential to prevent co-localization with the midgut epithelium and allow colonization without detrimental stimulation of an antibacterial immune response. UxuR, also found to have increased abundance in the parental strain $(\log 2 \mathrm{FC}=0.83)$, is a transcription factor that negatively regulates the expression of UidA, a key enzyme of the hexuronate metabolic pathway in Escherichia coli [54, 55]. Decreased expression of $u x u R$ in the passaged strain is therefore in line with the previous hypothesis that sugar transport may be involved in the selection process: a lower abundance of the $u x u R$ transcriptional repressor would allow greater utilization of sugar in the mosquito midgut as a nutrient source. sulA $(\log 2 \mathrm{FC}=0.90)$ is regulated by the SOS response, an inducible DNA repair mechanism, and halts cell division to prevent proliferation of DNA damage. The bacterial SOS response can be activated by exposure to antimicrobials and changes in oxidative conditions [56], conditions that prevail in the mosquito midgut. This hostile environment may cause upregulation of sulA in the parental strain and, conversely, lower expression in the passaged strain, since it is tolerant to the environment.
This microarray analysis has revealed that elevated expression of genes involved in response to the external environment likely contributes to the ability of the passaged Esp_Z strain to sense the local environment, and it is critical to the bacterium's ability to more effectively colonize the mosquito midgut. In addition, decreases in the abundance of transcripts involved in the SOS damage response and repression of sugar metabolism suggest that the selected strain is more suited to the hostile midgut environment.

\section{Transcriptomic survey for Esp_Z-encoded Plasmodium inhibition factors}

Previous experiments herein have shown that Esp_Z inhibits Plasmodium ookinete formation via a mechanism that involves bacterial production of an oxidative environment. This mechanism is likely controlled either directly or indirectly by a genetic component of the bacteria. Earlier studies also showed that $E s p \_Z$ produces factors that inhibit parasite development in vitro when cultured in an ookinete medium, but not when cultured under conditions of basic replication in LB broth [10]. To identify putative $E s p \_Z$ genes involved in the ROSmediated killing of Plasmodium parasites, the same whole-genome microarray approach to compare gene expression between $E s p \_Z$ grown in ookinete and LB media was employed, since only the ookinete medium induces ROS production [10]. A total of 98 and 61 genes enriched in ookinete medium- and LB medium-grown $E s p \_Z$ were identified, respectively (Fig. 4c; Additional file 2: Table S1). Sequences within the gene ontology groups that included oxidation-reduction process, transport, and respiratory electron transport chain were identified.

Genes of particular interest with increased abundance under ookinete medium conditions are summarized in Table 2. Upregulation of a transcript containing 
Table 2 ROS-producing candidate genes that are differentially regulated between Esp_Z $Z$ grown in ookinete medium conducive to ROS production, and LB medium

\begin{tabular}{llll}
\hline Gene ID & Description & Process GO category & Fold change (log $\mathbf{2})$ \\
\hline ESP_Z_1948 & GTP cyclohydrolase II & Riboflavin biosynthetic process & 1.384 \\
ESP_Z_1388 & NADPH nitroreductase & Oxidation-reduction process & 1.359 \\
ESP_Z_4666 & Molybdenum-pterin binding domain protein & Transmembrane transport & 1.169 \\
ESP_Z_1849 & Cytochrome b561 family protein & Respiratory electron transport chain & 1.105 \\
\hline
\end{tabular}

molybdenum-pterin binding domains was observed $(\log 2 \mathrm{FC}=1.17)$ and proteins with such domains are important co-factors for the acquisition of molybdenum. This trace element functions as a catalyst for molybdenum-dependent two-electron reduction-oxidation (redox) reactions [57], indicating that upregulation of this transcript in the ookinete medium-cultured bacteria may lead to the production of elevated ROS levels. GTP cyclohydrolase II $(\log 2 \mathrm{FC}=1.38)$ catalyses the first step of the biosynthesis pathway for riboflavin [58], an important precursor to bacterial redox sensors such as flavin mononucleotide (FMN) and flavin adenine dinucleotide (FAD) [59]. Elevated expression of this redox sensor precursor may suggest that ookinete medium-derived $E s p_{-} Z$ bacteria are responding to the presence of elevated ROS levels. We also observed increased abundance of a putative NADPH nitroreductase $(\log 2 \mathrm{FC}=1.36)$. Nitroreductases donate electrons to nitrogen-containing aromatic compounds generating ROS in the process [60], and this process has been suggested to mediate the trypanocidal activity of Nifurtimox and Benznidazole [61]. Interestingly, nitroreductases require either FMN or FAD as a co-factor for the generation of free radicals [62]: upregulation of both an NADPH nitroreductase and GTP cyclohydrolase II suggests that ookinete medium-cultured $E s p_{-} Z$ are generating ROS at least partially through the reduction of flavins.

In addition, a cytochrome b561, upregulated in the ookinete medium-grown $E s p \_Z(\log 2 \mathrm{FC}=1.11)$, has been shown to allow for regeneration of the reduced, ROS-detoxifying form of ascorbic acid [63]. It has previously been shown that supplementation with ascorbic acid or reduced glutathione and the resulting decreased redox environment limit the ability of $E s p_{-} Z$ to inhibit Plasmodium development [10]. Upregulation of a reducer of ascorbic acid suggests that the ookinete mediumderived $E s p \_Z$ bacteria are in an increased ROS environment and require an increased abundance of ascorbic acid in its reduced, active state. It becomes apparent that $E s p_{-} Z$, when grown in an environment conducive to ROS production, elevates transcripts associated with the generation of free radicals, and a combination of pathways are likely to contribute to the generation of anti-Plasmodium reactive species.

\section{Influence of Esp_Z on Plasmodium antioxidant gene expression}

We have previously shown that Esp_Z mediates Plasmodium killing through a ROS-dependent mechanism, as supplementation with antioxidants, such as ascorbic acid or reduced glutathione, limits the ability of Esp_Z to inhibit Plasmodium development [10]. In addition, since genes conducive to an elevated ROS environment are upregulated within this bacterium (Table 2), it lead to the hypothesis that the production of ROS may affect the expression of key Plasmodium antioxidant genes. To test this possibility, in vitro ookinete cultures of $P$. berghei were exposed to Esp_Z, a control anti-Plasmodium bacteria that does not produce ROS (Pseudomonas putida, $P p u$ ) [10], or to a PBS control, and the expression of five parasite antioxidant genes was measured over time: thioredoxin reductase (trxr), thioredoxin (trx), thioredoxin 1 (trx 1), peroxiredoxin-1 (tpx-1), and 1-Cys peroxiredoxin (1-cys-prx). Expression of tpx-1 and 1-cys prx have previously been shown to peak at 12 and $24 \mathrm{~h}$, respectively, after initiation of the ookinetes culture, and both increase in abundance in the presence of a superoxide producer; no impact on trx expression was detected [16]. Plasmodium berghei tpx-1 knockout lines have been shown to have reduced gametocyte numbers [64] and to exhibit increased numbers of immature oocysts and, consequently, a reduction in the number of sporozoites [65].

Pooling the results of two independent experiments (each including three biological replicates), we observed a reduction in transcript abundance of all targets $8 \mathrm{~h}$ after treatment with $E s p_{Z} Z$ (Fig. 5). It is important to understand that arrested parasite development becomes apparent shortly after $8 \mathrm{~h}$ of co-culture with $E s p \_Z$ [10]. For all genes, the transcript abundance did not alter significantly when the ookinete cultures were exposed to Pseudomonas putida, nor were there significant differences on antioxidant gene expression between exposure to the ROS-producing Esp_Z or this non-ROS inducing Pseudomonas putida. Of note, also, is the fact that the expression of 1-Cys-peroxiredoxin seemed to follow a 

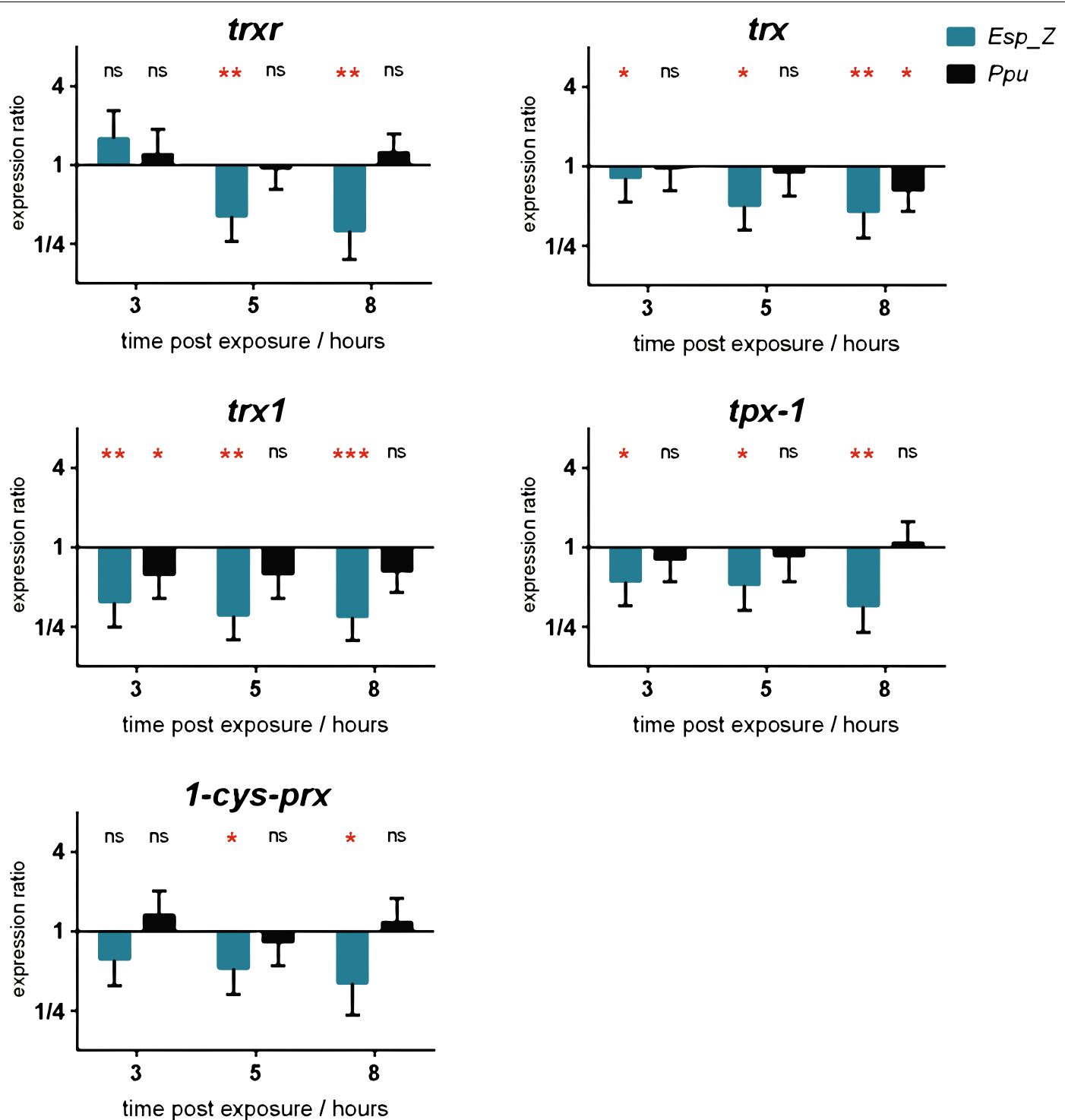

Fig. 5 Exposure to Esp_Z limits Plasmodium's antioxidant response. Relative P. berghei pre-ookinete expression of thioredoxin reductase (trxr), thioredoxin (trx), thioredoxin 1 (trx 1), peroxiredoxin-1 (tpx-1), and 1-Cys peroxiredoxin (1-cys-prx) upon in vitro co-culture with Esp_Z (blue) or Pseudomonas putida (Ppu, black). RT-qPCR datasets were normalized to the level of expression of 18SrRNA, a PBS control, and the expression at $1 \mathrm{~h}$ post-exposure for each experimental group. Bars represent the mean \pm the standard error of the mean of six biological replicates over two independent experiments $(3+3)$. Significance was determined using a two-tailed one sample t-test to determine whether each transcript was significantly up- or down-regulated $\left({ }^{*} p<0.05 ;{ }^{* *} p<0.01 ;{ }^{* * *} p<0.001\right)$

trend similar to that of the more canonical thioredoxin system enzymes, even though it has been suggested that in $P$. berghei 1-cys prx acts primarily to protect against heme-derived endogenous ROS, rather than exogenous ROS [66]. Taken together, these data indicate the possibility that Esp_Z interferes with ookinete maturation by partially and temporarily inducing a shutdown of the parasite's antioxidant response.

Plasmodium falciparum tpx-1 knockout lines display an increased sensitivity to the presence of superoxide producers [22], and expression of $P$. berghei tpx-1 and 1 -cys prx is increased in response to external ROS [16]. These results suggest that the downregulation of thioredoxin- 1 and the thioredoxin-dependent peroxiredoxins by $E s p \_Z$ renders the parasite more susceptible to the presence of Esp_Z-derived ROS [10]. P. berghei tpx-1 knockout lines demonstrate a reduced sporozoite load when compared to wild-type parasites [65], suggesting that the anti-parasite phenotype of $E s p_{-} Z$ may, in part, be due to a negative effect on the parasite's antioxidant defences. 


\section{Conclusions}

The devastating effects of malaria transmission are ever-present in sub-Saharan Africa, and increasing efforts to counteract these effects are either encountering resistance, as in the case of anti-malarial drugs and insecticides, or poor efficiencies, in the case of vaccine development. It is, therefore, imperative that alternate strategies be explored, and the microbiota of disease vectors has become an attractive resource for this purpose. Not only do certain members of the vector microbiota nearly eliminate Plasmodium infection $[9,10]$, but some members have also been shown to effectively kill the mosquito vectors of several pathogens [15]. Here, the potential of the naturally occurring Enterobacter species $E s p_{2} Z$ as part of a malaria control strategy was evaluated.

Proteobacteria are highly abundant in field-caught mosquitoes, including Enterobacter species, showing that they can be acquired and persist in the mosquito and its habitat $[7,8]$. In order to influence the natural microbiota, bacteria could be introduced via sugar-baited traps [41] thus ensuring Plasmodium exposure to them. It was demonstrated that $E s p \_Z$ can persist for up to four days after introduction and inhibit $P$. falciparum development when introduced via sugar meal (Fig. 1). As part of a translation study, it is important to examine the introduction and retention of bacteria in the adult midgut, because of the as-yet unclear data on transmission of bacteria from larvae to adults [67-69].

The impact on mosquito fitness of an introduced bacterium is an additional consideration, since a potentially negative impact is likely to be selected against in nature. Unlike the intracellular Wolbachia species, bacteria that are able to manipulate the host's reproductive system and thereby ensure vertical dissemination through the population [70,71], the spread of a bacterium must not induce any fitness cost. Microbiota are important for mosquito fitness, with the proteobacteria Asaia sp. influencing larval development and several other species having been shown to affect mosquito fitness $[9,72]$. Naturally occurring bacterial isolates have a varying ability to persist and replicate when re-introduced into aseptic An. gambiae midguts [9], suggesting that there is variation in the adaptation to the mosquito midgut environment. This study has shed light on candidate molecular components that are important for bacterial colonization and persistence in the mosquito. It is possible that a combinatorial approach could be undertaken, utilizing a cocktail of anti-parasitic bacteria, each having a different anti-parasitic mechanism, for the control of malaria transmission.

In the present study, the nature of the anti-Plasmodium activity of Esp_Z was also further characterized. On the one hand, the observation that Esp_Z upregulates genes associated with the generation of ROS when cultured under conditions shown to inhibit parasite development in vitro was validated. This finding corroborates previous studies in which $E s p \_Z$ was shown to induce an oxidative environment when co-cultured with Plasmodium ookinetes, with such an environment being the likely cause of parasite inhibition. On the other hand, a potential complementary mechanism by which the bacteria appears to directly interfere with the parasite's antioxidant system was uncovered, thereby limiting its ability to respond and adapt to the oxidative environment it promotes. This mechanism is indicated by the downregulation of key genes of the thioredoxin and glutathione antioxidant pathways in P. berghei ookinetes, until the moment when parasite development is arrested. Current efforts are underway to identify putative mediators of this effect in order to further characterize this phenomenon, in what also constitutes an exploration of Esp_Z's metabolome from a natural product discovery perspective, in the search for new and innovative anti-malarials.

\section{Additional files}

\begin{abstract}
Additional file 1: Figure S1. Impact of blood feeding Esp_Z on mosquito fitness. (A) Longevity studies were performed following blood-meal introduction of either Esp_Z, bacterial cocktail, or PBS into aseptic mosquito cohorts. A sterile blood meal was provided on day 4 (black arrow), and unfed mosquitoes were censored from the analysis. Survival was monitored daily and continued until $100 \%$ mortality was reached. The curves represent the average percent mortality across three replicates, and the error bars indicate the standard error. Significance was determined using the log-rank test (Mantel-Cox) using a Kaplan-Meier survival analysis. (B) Fecundity analysis between Esp_Z-, bacterial cocktailand PBS-fed blood-fed mosquito cohorts. Separate cohorts were provided a second blood meal $72 \mathrm{~h}$ after blood-meal introduction of Esp_Z, and circles represent the number of eggs laid per female. Horizontal bars represent the median number of eggs, error bars indicate the standard error, and three pooled biological replicates are shown. Significance was determined using the Mann-Whitney test. (C) Fertility analysis in Esp_Z-, bacterial cocktail-, and PBS-fed blood-fed mosquitoes. At $72 \mathrm{~h}$ after introduction, mosquitoes were offered a second, naive blood meal, and those not engorged were removed. Eggs were collected $48 \mathrm{~h}$ post-blood meal and allowed to hatch in rearing trays. The hatch rate indicates the percentage of eggs giving rise to 1st instar larvae; the error bars indicate the standard error of the mean, and significance was determined using an unpaired $t$-test
\end{abstract}

Additional file 2: Table S1. Global results of DNA microarray analysis of Esp_Z transcriptome upon selection in the mosquito midgut environment (P1 vs. P3) and upon growth in Plasmodium inhibiting conditions (Ook vs. $\mathrm{LB})$.

\footnotetext{
Abbreviations

ROS: reactive oxygen species; CFU: colony forming units; Esp_Z: Enterobacter sp. Zambiae; IGS: Institute for Genome Sciences; Log2FC: binary logarithm of fold change; redox: reduction-oxidation; FMN: flavin mononucleotide; FAD: flavin adenine dinucleotide; Tpx-1: peroxiredoxin-1; 1-Cys-prx: 1-cysperoxiredoxin; Trx-1: thioredoxin-1; trxr: thioredoxin reductase; trx: thioredoxin; trx1: thioredoxin 1; tpx-1: peroxiredoxin-1; 1-cys-prx: 1-Cys peroxiredoxin; $\mathrm{TP}_{\mathrm{G}}$ : peroxidase-like thioredoxin peroxidase.
} 


\section{Authors' contributions}

NJD, RGS, CMC, GD conceived experiments. NJD, RGS, CMC, EFM, GM performed experiments. NJD, RGS, CMC, EFM, GD analysed data obtained. NJD, RGS, CMC, EFM, GD wrote the manuscript. All authors read and approved the final manuscript.

\section{Author details}

${ }^{1}$ W. Harry Feinstone Department of Molecular Microbiology and Immunology, Bloomberg School of Public Health, Johns Hopkins University, Baltimore, MD, USA. ${ }^{2}$ Institute for Genome Sciences, University of Maryland School of Medicine, Baltimore, MD, USA.

\section{Acknowledgements}

We would like to thank the Johns Hopkins Malaria Research Institute Insectary and Parasitology core facilities.

\section{Competing interests}

The authors declare that they have no competing interests.

\section{Availability of data and material}

Esp_Z genome is deposited in NCBI with BioSample accession no. SAMN05525567.

\section{Consent for publication}

This manuscript does not include details, images, or videos relating to individual participants that would otherwise require written informed consent for the publication.

\section{Ethics approva}

This study was carried out in strict accordance with the recommendations in the Guide for the Care and Use of Laboratory Animals of the National Institutes of Health. Mice were only used for mosquito rearing as a blood source, according to approved protocol. The protocol was approved by the Animal Care and Use Committee of the Johns Hopkins University (Permit Number: M006H300). Commercial anonymous human blood from Interstate Blood Bank was used for Plasmodium infection assays in mosquitoes, and informed consent was therefore not applicable. The Johns Hopkins School of Public Health Ethics Committee has approved this protocol.

\section{Funding}

This work has been supported by NIH/NIAID Grants AI081877, Al061576, a Lang family fellowship (to NJD), a Boehringer Ingelheim Fonds fellowship (to RGS) and a Johns Hopkins Malaria Research Institute Fellowship (to CMC).

Received: 7 May 2016 Accepted: 2 August 2016

Published online: 22 August 2016

\section{References}

1. World Health Organization. World Malaria Report 2015. Geneva: World Health Organization; 2016

2. Ashley EA, Dhorda M, Fairhurst RM, Amaratunga C, Lim P, Suon S, et al. Spread of artemisinin resistance in Plasmodium falciparum malaria. N Engl J Med. 2014;371:411-23.

3. Knox TB, Juma EO, Ochomo EO, Jamet HP, Ndungo L, Chege P, et al. An online tool for mapping insecticide resistance in major Anopheles vectors of human malaria parasites and review of resistance status for the Afrotropical region. Parasit Vectors. 2014;7:14

4. Saraiva RG, Kang S, Simões ML, Angleró-Rodríguez Yl, Dimopoulos G. Mosquito gut antiparasitic and antiviral immunity. Dev Comp Immunol. 2016;64:53-64.

5. Smith RC, Vega-Rodríguez J, Jacobs-Lorena M. The Plasmodium bottleneck: malaria parasite losses in the mosquito vector. Mem Instituto Oswaldo Cruz. 2014;109:644-61.

6. Dennison NJ, Jupatanakul N, Dimopoulos G. The mosquito microbiota influences vector competence for human pathogens. Curr Opin Insect Sci. 2014;3:6-13.

7. Boissiere A, Tchioffo MT, Bachar D, Abate L, Marie A, Nsango SE, et al. Midgut microbiota of the malaria mosquito vector Anopheles gambiae and interactions with Plasmodium falciparum infection. PLoS Pathog. 2012;8:e1002742

8. Osei-Poku J, Mbogo CM, Palmer WJ, Jiggins FM. Deep sequencing reveals extensive variation in the gut microbiota of wild mosquitoes from Kenya. Mol Ecol. 2012;21:5138-50.

9. Bahia AC, Dong Y, Blumberg BJ, Mlambo G, Tripathi A, BenMarzoukHidalgo OJ, et al. Exploring Anopheles gut bacteria for Plasmodium blocking activity. Environ Microbiol. 2014;16:2980-94.

10. Cirimotich CM, Dong YM, Clayton AM, Sandiford SL, Souza-Neto JA, Mulenga $\mathrm{M}$, et al. Natural microbe-mediated refractoriness to Plasmodium infection in Anopheles gambiae. Science. 2011;332:855-8.

11. Dong YM, Aguilar R, Xi ZY, Warr E, Mongin E, Dimopoulos G. Anopheles gambiae immune responses to human and rodent Plasmodium parasite species. PLoS Pathog. 2006;2:e52.

12. Bando H, Okado K, Guelbeogo WM, Badolo A, Aonuma H, Nelson B, et al. Intra-specific diversity of Serratia marcescens in Anopheles mosquito midgut defines Plasmodium transmission capacity. Sci Rep. 2013;3:1641.

13. Gonzalez-Ceron L, Santillan F, Rodriguez MH, Mendez D, Hernandez-Avila JE. Bacteria in midguts of field-collected Anopheles albimanus block Plasmodium vivax sporogonic development. J Med Entomol. 2003:40:371-4.

14. Rodrigues J, Brayner FA, Alves LC, Dixit R, Barillas-Mury C. Hemocyte differentiation mediates innate immune memory in Anopheles gambiae mosquitoes. Science. 2010;329:1353-5.

15. Ramirez JL, Short SM, Bahia AC, Saraiva RG, Dong Y, Kang S, et al. Chromobacterium Csp_P reduces malaria and dengue infection in vector mosquitoes and has entomopathogenic and in vitro anti-pathogen activities. PLoS Pathog. 2014;10:e1004398.

16. Turturice BA, Lamm MA, Tasch JJ, Zalewski A, Kooistra R, Schroeter EH, et al. Expression of cytosolic peroxiredoxins in Plasmodium berghei ookinetes is regulated by environmental factors in the mosquito bloodmeal. PLoS Pathog. 2013;9:e1003136.

17. Jortzik E, Becker K. Thioredoxin and glutathione systems in Plasmodium falciparum. Int J Med Microbiol. 2012;302:187-94.

18. Molina-Cruz A, Dejong RJ, Charles B, Gupta L, Kumar S, Jaramillo-Gutierrez $\mathrm{G}$, et al. Reactive oxygen species modulate Anopheles gambiae immunity against bacteria and Plasmodium. J Biol Chem. 2008;283:3217-23.

19. Muller S. Redox and antioxidant systems of the malaria parasite Plasmodium falciparum. Mol Microbiol. 2004:53:1291-305.

20. Sztajer H, Gamain B, Aumann KD, Slomianny C, Becker K, Brigelius-Flohe R, et al. The putative glutathione peroxidase gene of Plasmodium falciparum codes for a thioredoxin peroxidase. J Biol Chem. 2001;276:7397-403.

21. Sturm N, Jortzik E, Mailu BM, Koncarevic S, Deponte M, Forchhammer K, et al. Identification of proteins targeted by the thioredoxin superfamily in Plasmodium falciparum. PLoS Pathog. 2009:5:e1000383.

22. Komaki-Yasuda K, Kawazu S, Kano S. Disruption of the Plasmodium falciparum 2-Cys peroxiredoxin gene renders parasites hypersensitive to reactive oxygen and nitrogen species. FEBS Lett. 2003;547:140-4.

23. Haselton KJ, David R, Fell K, Schulte E, Dybas M, Olsen KW, et al. Molecular cloning, characterization and expression profile of a glutathione peroxidase-like thioredoxin peroxidase (TPxGl) of the rodent malaria parasite Plasmodium berghei. Parasitol Int. 2015;64:282-9.

24. Munigunti R, Gathiaka S, Acevedo O, Sahu R, Tekwani B, Calderon Al. Characterization of PfTrxR inhibitors using antimalarial assays and in silico techniques. Chem Cent J. 2013;7:175.

25. Gary RE, Foster WA. Effects of available sugar on the reproductive fitness and vectorial capacity of the malaria vector Anopheles gambiae (Diptera: culicidae). J Med Entomol. 2001;38:22-8.

26. Favia G, Dimopoulos G, Dellatorre A, Toure YT, Coluzzi M, Louis C. Polymorphisms detected by random PCR distinguish between different chromosomal forms of Anopheles gambiae. Proc Natl Acad Sci USA. 1994;91:10315-9.

27. Pfaff MW. A new mathematical model for relative quantification in realtime RT-PCR. Nucleic Acids Res. 2001;29:e45.

28. Angiuoli SV, Matalka M, Gussman A, Galens K, Vangala M, Riley DR, et al. ClovR: a virtual machine for automated and portable sequence analysis from the desktop using cloud computing. BMC Bioinformatics. 2011;12:356.

29. Aguilar R, Simard F, Kamdem C, Shields T, Glass GE, Garver LS, et al. Genome-wide analysis of transcriptomic divergence between laboratory colony and field Anopheles gambiae mosquitoes of the $\mathrm{M}$ and S molecular forms. Insect Mol Biol. 2010;19:695-705. 
30. Sim S, Jupatanakul N, Ramirez JL, Kang S, Romero-Vivas CM, Mohammed $\mathrm{H}$, et al. Transcriptomic profiling of diverse Aedes aegypti strains reveals increased basal-level immune activation in dengue virus-refractory populations and identifies novel virus-vector molecular interactions. PLOS Negl Trop Dis. 2013;7:e2295.

31. Herrero J, Al-Shahrour F, Diaz-Uriarte R, Mateos A, Vaquerizas JM, Santoyo $J$, et al. GEPAS: a web-based resource for microarray gene expression data analysis. Nucleic Acids Res. 2003;31:3461-7.

32. Dudoit S, Gentleman RC, Quackenbush J. Open source software for the analysis of microarray data. Biotechniques. 2003;34(13):45-51.

33. Yang IV, Chen E, Hasseman JP, Liang W, Frank BC, Wang S, et al. Within the fold: assessing differential expression measures and reproducibility in microarray assays. Genome Biol. 2002;3:0062.

34. Rani A, Sharma A, Rajagopal R, Adak T, Bhatnagar RK. Bacterial diversity analysis of larvae and adult midgut microflora using culture-dependent and culture-independent methods in lab-reared and field-collected Anopheles stephensi-an Asian malarial vector. BMC Microbiol. 2009;9:96.

35. Nayar JK. Vanhande.E. The fuel for sustained mosquito flight. J Insect Physiol. 1971;17:471-81.

36. Foster WA. Mosquito sugar feeding and reporoductive energetics. Annu Rev Entomol. 1995;40:443-74.

37. Quinones ML, Lines JD, Thomson MC, Jawara M, Morris J, Greenwood BM. Anopheles gambiae gonotrophic cycle duration, biting and exiting behaviour unaffected by permethrin-impregnated bednets in The Gambia. Med Vet Entomol. 1997;11:71-8.

38. Morrow R, Moss W. The Epidemiology and Control of Malaria. In: Nelson KE, Williams C, editors. Infectious disease epidemiology. 3rd ed. Burlington: Jones \& Bartlett Learning; 2014.

39. Wang Y, Gilbreath TM, Kukutla P, Yan G, Xu J. Dynamic gut microbiome across life history of the malaria mosquito Anopheles gambiae in Kenya. PLOS ONE. 2011;6:e24767.

40. Minard G, Mavingui P, Moro CV. Diversity and function of bacterial microbiota in the mosquito holobiont. Parasit Vectors. 2013:6:146.

41. Muller GC, Beier JC, Traore SF, Toure MB, Traore MM, Bah S, et al. Successful field trial of attractive toxic sugar bait (ATSB) plant-spraying methods against malaria vectors in the Anopheles gambiae complex in Mali, West Africa. Malar J. 2010:9:210

42. Gouagna LC, Mulder B, Noubissi E, Tchuinkam T, Verhave JP, Boudin C. The early sporogonic cycle of Plasmodium falciparum in laboratory-infected Anopheles gambiae: an estimation of parasite efficacy. Trop Med Int Health. 1998;3:21-8.

43. Riehle MA, Moreira CK, Lampe D, Lauzon C, Jacobs-Lorena M. Using bacteria to express and display anti-Plasmodium molecules in the mosquito midgut. Int J Parasitol. 2007;37:595-603.

44. Hueck CJ. Type III protein secretion systems in bacterial pathogens of animals and plants. Microbiol Mol Biol Rev. 1998;62:379-433.

45. Fennelly NK, Sisti F, Higgins SC, Ross PJ, van der Heide H, Mooi FR, et al. Bordetella pertussis expresses a functional type III secretion system that subverts protective innate and adaptive immune responses. Infect Immun. 2008;76:1257-66.

46. Correa VR, Majerczak DR, Ammar ED, Merighi M, Pratt RC, Hogenhout SA, et al. The bacterium Pantoea stewartii uses two different type III secretion systems to colonize its plant host and insect vector. Appl Environ Microbiol. 2012;78:6327-36.

47. Polissi A, Pontiggia A, Feger G, Altieri M, Mottl H, Ferrari L, et al. Largescale identification of virulence genes from Streptococcus pneumoniae. Infect Immun. 1998;66:5620-9.

48. Song F, Peng Q, Brillard J, Buisson C, de Been M, Abee T, et al. A multicomponent sugar phosphate sensor system specifically induced in Bacillus cereus during infection of the insect gut. FASEB J. 2012;26:3336-50.

49. Ryter SW, Tyrrell RM. The heme synthesis and degradation pathways: role in oxidant sensitivity. Heme oxygenase has both pro- and antioxidant properties. Free Radic Biol Med. 2000;28:289-309.

50. Oliveira JHM, Goncalves RLS, Lara FA, Dias FA, Gandara ACP, MennaBarreto RFS, et al. Blood meal-derived heme decreases ROS levels in the midgut of Aedes aegypti and allows proliferation of intestinal microbiota. PLoS Pathog. 2011;7:e1001320.
51. Bliska JB, Copass MC, Falkow S. The Yersinia pseudotuberculosis adhesin YadA mediates intimate bacterial attachment to and entry into HEp-2 cells. Infect Immun. 1993:61:3914-21.

52. Leo JC, Elovaara H, Bihan D, Pugh N, Kilpinen SK, Raynal N, et al. First analysis of a bacterial collagen-binding protein with collagen Toolkits: promiscuous binding of YadA to collagens may explain how YadA interferes with host processes. Infect Immun. 2010;78:3226-36.

53. Kumar S, Molina-Cruz A, Gupta L, Rodrigues J, Barillas-Mury C. A peroxidase/dual oxidase system modulates midgut epithelial immunity in Anopheles gambiae. Science. 2010;327:1644-8.

54. Blanco C. Transcriptional and translational signals of the vidA gene in Escherichia coli-K12. Mol Gen Genet. 1987;208:490-8.

55. Novel M, Novel G. Regulation of beta-glucuronidase synthesis in Escherichia coli K-12: pleiotropic constitutive mutations affecting $u \times u$ and uidA expression. J Bacteriol. 1976;127:418-32.

56. Poole K. Bacterial stress responses as determinants of antimicrobial resistance. J Antimicrob Chemother. 2012;67:2069-89.

57. Schwarz G, Mendel RR, Ribbe MW. Molybdenum cofactors, enzymes and pathways. Nature. 2009;460:839-47.

58. Foor F, Brown GM. Purification and properties of guanosine triphosphate cyclohydrolase II from Eschericia coli. J Biol Chem. 1975;250:3545-51.

59. Green J, Paget MS. Bacterial redox sensors. Nat Rev Microbiol. 2004;2:954-66

60. Peterson FJ, Mason RP, Hovsepian J, Holtzman JL. Oxygen-sensitive and oxygen-insensitive nitroreduction by Escherichia coli and rat hepatic microsomes. J Biol Chem. 1979;254:4009-14.

61. Hall BS, Bot C, Wilkinson SR. Nifurtimox activation by trypanosomal type I nitroreductases generates cytotoxic nitrile metabolites. J Biol Chem. 2011;286:13088-95.

62. Watanabe M, Nishino T, Takio K, Sofuni T, Nohmi T. Purification and characterization of wild-type and mutant "classical" nitroreductases of Salmonella typhimurium - L33R mutation greatly diminishes binding of FMN to the nitroreductase of S. typhimurium. J Biol Chem. 1998:273:23922-8.

63. Njus D, Kelley PM, Harnadek GJ, Pacquing YV. Mechanism of ascorbic acid regeneration mediated by cytochrome b561. Ann NY Acad Sci. 1987:493:108-19.

64. Yano K, Komaki-Yasuda K, Tsuboi T, Torii M, Kana S, Kawazu S. 2-Cys peroxiredoxin TPX-1 is involved in gametocyte development in Plasmodium berghei. Mol Biochem Parasitol. 2006;148:44-51.

65. Yano K, Otsuki H, Arai M, Komaki-Yasuda K, Tsuboi T, Torii M, et al. Disruption of the Plasmodium berghei 2-Cys peroxiredoxin TPX-1 gene hinders the sporozoite development in the vector mosquito. Mol Biochem Parasitol. 2008;159:142-5.

66. Kawazu S, Ikenoue N, Takemae H, Komaki-Yasuda K, Kano S. Roles of 1-Cys peroxiredoxin in haem detoxification in the human malaria parasite Plasmodium falciparum. FEBS J. 2005;272:1784-91.

67. Pumpuni CB, DeMaio J, Kent M, Davis JR, Beier JC. Bacterial population dynamics in three anopheline species: the impact on Plasmodium sporogonic development. Am J Trop Med Hyg. 1996;54:214-8.

68. Lindh JM, Borg-Karlson AK, Faye I. Transstadial and horizontal transfer of bacteria within a colony of Anopheles gambiae (Diptera: Culicidae) and oviposition response to bacteria-containing water. Acta Trop. 2008:107:242-50.

69. Moll RM, Romoser WS, Modrzakowski MC, Moncayo AC, Lerdthusnee K. Meconial peritrophic membranes and the fate of midgut bacteria during mosquito (Diptera: culicidae) metamorphosis. J Med Entomol. 2001:38:29-32.

70. Xi ZY, Khoo CCH, Dobson SL. Wolbachia establishment and invasion in an Aedes aegypti laboratory population. Science. 2005;310:326-8.

71. Bian GW, Joshi D, Dong YM, Lu P, Zhou GL, Pan XL, et al. Wolbachia invades Anopheles stephensi populations and induces refractoriness to Plasmodium infection. Science. 2013;340:748-51.

72. Chouaia B, Rossi P, Epis S, Mosca M, Ricci I, Damiani C, et al. Delayed larval development in Anopheles mosquitoes deprived of Asaia bacterial symbionts. BMC Microbiol. 2012;12(Suppl 1):S2. 